\title{
Correction to: Redistribution to the less productive: parallel characterizations of the egalitarian Shapley and consensus values
}

\author{
Koji Yokote ${ }^{1}$ (D) Takumi Kongo $^{2} \cdot$ Yukihiko Funaki $^{3}$ \\ Published online: 21 December 2020 \\ (C) Springer Science+Business Media, LLC, part of Springer Nature 2020
}

\section{Correction to: Theory and Decision https://doi.org/10.1007/s11238-020-09781-1}

In Sect. 3.3, the terms "one-person" and "(n-1)-person" were incorrectly updated by mistake during the correction stage in the online published article. The correct sentences are given below:

Proportionately decreasing redistribution in one-person unanimity games $\left(\mathrm{PDR}^{1}\right)$ : For any $\left(N, u_{i}\right),\left(M, u_{j}\right) \in G$, with $i, k \in N, i \neq k, 2 \leq|N|, j, \ell \in M, j \neq \ell$, and $2 \leq|M|,|N| f_{k}\left(N, u_{i}\right)=|M| f_{\ell}\left(M, u_{j}\right)$.

Proportionately decreasing redistribution in $(n-1)$-person unanimity games $\left(\mathrm{PDR}^{n-1}\right)$ : For any $\left(N, u_{N \backslash i}\right),\left(M, u_{M \backslash j}\right) \in G$, with $i \in N, 3 \leq|N|, j \in M$, and $3 \leq|M|,|N| f_{i}\left(N, u_{N \backslash i}\right)=|M| f_{j}\left(M, u_{M \backslash j}\right)$.

The original article has been corrected accordingly.

We apologize for the error introduced during the production process.

The original article can be found online at https://doi.org/10.1007/s11238-020-09781-1.

Takumi Kongo

kongo@adm.fukuoka-u.ac.jp

Koji Yokote

sidehand@toki.waseda.jp

Yukihiko Funaki

funaki@waseda.jp

1 Waseda Institute for Advanced Study, Waseda University, 1-21-1 Nishi-Waseda, Shinjuku-ku, Tokyo 169-0051, Japan

2 Faculty of Economics, Fukuoka University, 8-19-1 Nanakuma, Jonan-ku, Fukuoka 814-0180, Japan

3 Faculty of Political Science and Economics, Waseda University, 1-6-1, Nishi-Waseda, Shinjuku-ku, Tokyo 169-8050, Japan 